\section{Onychoheterotopia in children}

\author{
Marco Ferrari, ${ }^{1,2}$ Vittorio Mazzarello, ${ }^{2}$ \\ Egidio Barbi ${ }^{1,3}$ \\ ${ }^{1}$ Pediatric Department, University of \\ Trieste; ${ }^{2}$ Skinlab, Department of \\ Biomedical Sciences, University of \\ Sassari; ${ }^{3}$ Institute for Maternal and \\ Child Health IRCCS, Burlo Garofolo of \\ Trieste, Italy
}

\begin{abstract}
The ectopic nail (EN) is an additional nail located in an abnormal site. It belongs to the onycho-heterotopia, a rare condition whose pathogenesis is indeterminate. This article illustrates the clinical-morphological and dermoscopic points of view, the diagnostic criteria, the possible pathogenesis, and surgical treatment of this pediatric onycho-heterotopia.
\end{abstract}

\section{Introduction}

Ectopic nail (EN) is an additional and independent nail located in an abnormal site. Also known as onycho-heterotopia, it is an extremely rare condition whose pathogenesis remains undetermined. ${ }^{1} \mathrm{EN}$ and most of the nail diseases, which appear in the first decade of life, are congenital; less frequently, EN is described as acquired or post-traumatic. Onychoheterotopia is a single lesion involving the sole or, in most of the reported cases, occurring along with the regular fingernail, on the distal phalanx, and is attributed to digital malformations (e.g., palmar nail syndrome) or bone alterations. ${ }^{2}$ The appearance of the anomalous nail at diagnosis ranges from 2 to 13 years, and females are more affected than males.

\section{Case Report}

An 11-year-old girl, skin phototype 3 of Fitzpatrick, was evaluated for an EN lesion on the right sole. The patient complained of occasional pains while walking and dancing. Family history was not significant for genodermatosis or nail disorders. The lesion appeared at the age of 4 and had slowly increased since then. It was recently diagnosed as a wart and treated with cryotherapy without any benefit.

The clinical presentation showed cylindrical-conical keratotic horns on admission
(Figure 1). The surrounding skin was smooth, with no signs of swelling or inflammation. We performed a dermoscopic analysis, which revealed a surface made irregular by transverse grooves at the periungual sulcus base (Figure 2). Treatment of EN consists of its removal. EN was treated under local anesthesia by surgical excision followed by phenol's application on the nail matrix. No local recurrence was observed in the two-years follow-up.

\section{Discussion}

The onycho-heterotopia is a single lesion that usually appears near the fingers and rarely involves the feet. ${ }^{3}$ The clinical features are dependent on the nail tissue's anatomical site, and occasional pain may be reported. Histopathological and ultrastructural features are similar to those of a regular nail with a reverse growth pattern. ${ }^{4}$ Three zones constitute EN: nail matrix, nail bed, nail plate, similar onycholysis, but differently arranged compared to a standard nail. ${ }^{5}$ The EN has a matrix in the center or back, with a horizontal-vertical growth pattern and a distal or inverted orientation. The neo-nail is generally conical and spatulashaped at dermoscopic presentation, looking like a rudimentary fingernail with a corrugated surface and transverse furrows. ${ }^{3}$ The right foot's little finger is the most commonly involved in EN, followed by the 4th digit and the 2nd digit. Several hypotheses tried to explain the origin of EN: the traumatic inoculation of onycholysis or implantation of nail germ cells and their abnormal growth; ${ }^{6}$ the variation in the long arm of chromosome 6 with congenital polyonychia $;{ }^{7}$ the presence of stray germinal cells in ectopic sites; ${ }^{8}$ the phenomenon of "paleotenia" $($ paleo $=$ ancient; te1'v $=$ to verge on $)$ meaning the appearance of a primitive silent character, present in other animal species having a common phylogenetic origin. The pathogenesis remains unclear; it seems most probable that ectopic nails are related to the alterations of particular genes responsible for nail growth and orientation. ${ }^{9}$ The EN is often confused with acquired digital fibroma, cutaneous horn, clavus, foreign body, split nail deformity, rudimentary polydactyly associated with callus formation and warts, and treated accordingly. The lesion is often undiagnosed or misdiagnosed, and cryotherapy alone often results in relapses. Surgical treatment should be followed by the phenolization of the nail matrix so that the germinal matrix is completely removed and destroyed.
Correspondence: Marco Ferrari, Pediatrics, University of Trieste, Trieste 34137, Italy. Tel: +39.0403785454, Fax +39.0403785452

E-mail: dr.marcoferrari@gmail.com

Key words: Ectopic nail, Histopathology, Nail morphology, Nail dysplasias

Acknowledgments: The authors thank Martina Bradaschia for the English revision of the manuscript

Contributions: MF, Substantial contributions to the study, including conceptualization/ design, methodology, investigation, and formal analysis. Participation in writing and revising the manuscript. VM, Substantial contributions to the study, including conceptualization/design, methodology, investigation, supervision, data curation, and formal analysis. Participation in writing and revising the manuscript. EB, Substantial contributions to the study, including conceptualization/design, methodology, investigation, supervision, data curation, and resources. Participation in writing and revising the manuscript.

Conflict of interest: The authors declare no potential conflict of interest.

Funding: None.

Ethics approval: The study was approved by the local ethical committee.

Consent to publication: It was obtained by the research investigator.

Please cite this article as: Ferrari $M$, Mazzarello V, Barbi E. Onychoheterotopia in children. Dermatol Rep 2021;13:9068.

Received for publication: 6 January 2021. Revision received: 19 January 2021. Accepted for publication: 28 January 2021

This work is licensed under a Creative Commons Attribution-NonCommercial 4.0 International License (CC BY-NC 4.0).

(C) Copyright: the Author(s), 2021

Licensee PAGEPress, Italy

\section{Conclusions}

This report describes an unusual localization of a pediatric ectopic nail. Although this is a rare condition, its prompt recognition can avoid unnecessary treatments and allows appropriate surgical treatment without relapses. 


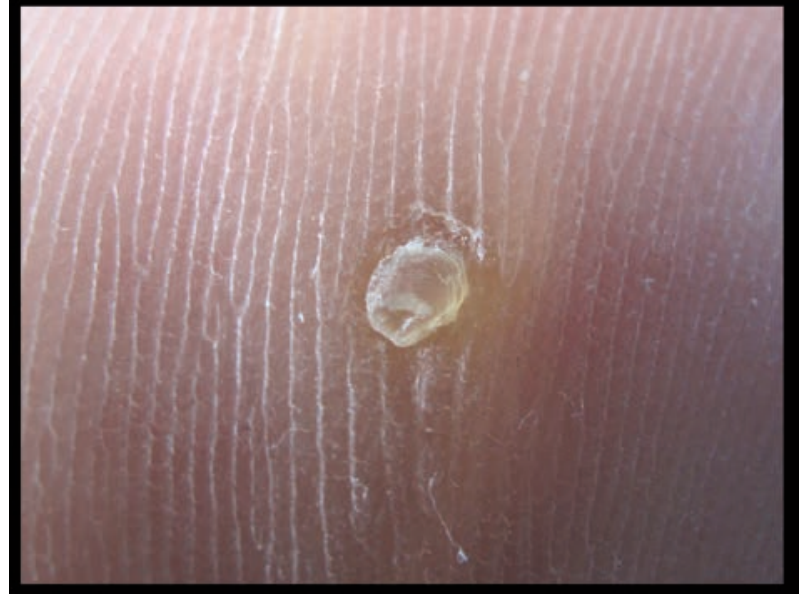

Figure 1. Clinical appearance of ectopic foot nails in an 11-year-old girl.

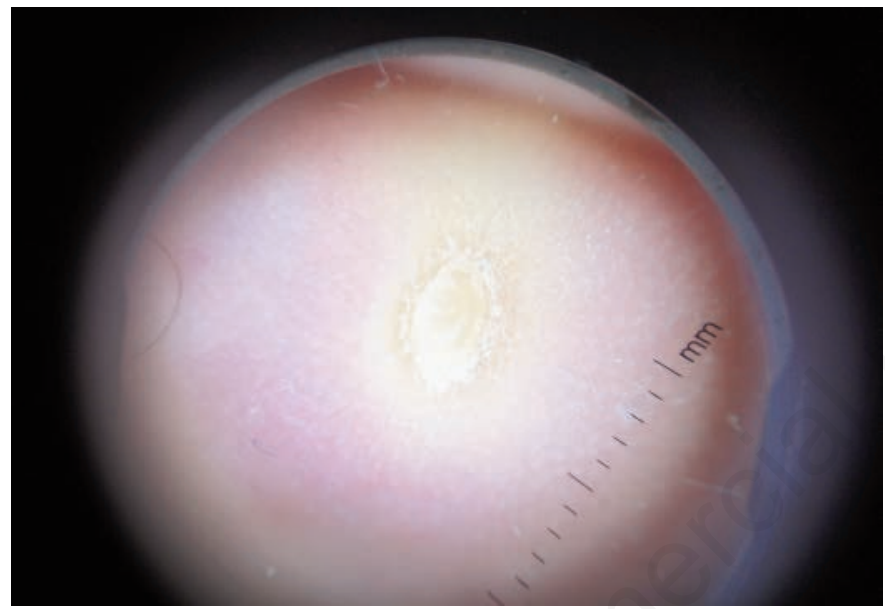

Figure 2. Dermoscopic features of the ectopic nail.

\section{References}

1. Riaz F, Rashid RM, Khachemoune A. Onychoheterotopia: pathogenesis, presentation, and management of ectopic nai. J Am Acad Dermatol 2011;64:1616.

2. Yadav S, Khullar G, Dogra S Congenital onychoheterotopia involving multiple toe nails. J Am Pediatr Med Assoc 2013;103:445-7.

3. Oiso N, Kurokawa I, Tsuruta D, et al. The histopathological feature of the nail isthmus in an ectopic nail. Am J Dermatopathol 2011;33:841-4.

4. Ena P, Ena L, Ferrari M, Mazzarello V. Ectopic Foot Nails: Clinical and dermoscopic features, treatment and outcome in 20 cases. Dermatology 2015;231:298-303.

5. Ena P, Enal L. Ferrari M, Mazzarello V. Double little toenails: report of 4 familial cases. JAAD Case Reports 2020;4,365-8.

6. Park JH, Kim JH, Lee JH, et al. Onychodermis (specialized nail mesenchyme) is present in ectopic nails. $\mathrm{J}$ Cutan Pathol 2013;40:600-2.

7. Soligo C, Muller AE. Nails and claws in primate evolution. J Hum Evol 1999;36:97-114.

8. Kopera D, Soyer HP, Kerl H. Ectopic calcaneal nail. J Am Acad Dermatol 1996;35:484-5.

9. Ena P, Mazzarello V, Dessy LA. Ectopic plantar nail: a report of two cases. Br J Dermatol 2003;149:1071-4. 